\title{
Simulation of Left Ventricle Flow Dynamics with Dilated Cardiomyopathy during the Filling Phase*
}

\author{
B. T. Chan, C. W. Ong, E. Lim, N. A. Abu Osman, A. Al Abed, N. H. Lovell and S. Dokos.
}

\begin{abstract}
Dilated cardiomyopathy (DCM) is a common cardiac disease which leads to the deterioration in cardiac performance. A computational fluid dynamics (CFD) approach can be used to enhance our understanding of the disease, by providing us with a detailed map of the intraventricular flow and pressure distributions. In the present work, effect of ventricular size on the intraventricular flow dynamics and intraventricular pressure gradients (IVPGs) was studied using two different implementation methods, i.e. the geometry-prescribed and the fluid structure interaction (FSI) methods. Results showed that vortex strength and IVPGs are significantly reduced in a dilated heart, leading to an increased risk of thrombus formation and impaired ventricular filling. We suggest FSI method as the ultimate method in studying ventricular dysfunction as it provides additional cardiac disease prognostic factors and more realistic model implementation.
\end{abstract}

\section{INTRODUCTION}

Dilated cardiomyopathy (DCM) is one of the most common cardiac diseases characterized by ventricular dilatation (end diastolic volume $>250 \mathrm{~mL}$ ) and myocardial contractile dysfunction [1]. The measureable functional deficiencies of DCM include low ejection fraction and decreased wall motion. Magnetic resonance imaging (MRI) and echocardiography are normally used to assess the severity of DCM through flow and geometrical measurements, such as end diastolic volume, end systolic volume and wall thickness.

Despite the rapid advancement of medical imaging modalities, they are restricted to global geometrical and flow measurements. The local hemodynamics indices, such as the spatial and temporal pressure distributions, wall shear stress, boundary flow layer and stagnation flow, which are important parameters for the early detection of heart failure [2], are beyond the scope of the imaging modalities provision. Experimental studies have shown that variations of flow distributions and vortex dynamics in the ventricle serve as potential cardiac health indicators $[3,4]$.

To overcome this problem, a computational fluid dynamic (CFD) approach, which provides spatial and temporal distributions of the velocity and pressure in the heart, has emerged as a reliable tool in enhancing our understanding of the pathophysiology and progression of the disease. Extensive studies have been carried out to investigate the flow distributions in the ventricle using two main types of CFD

*Research supported by MOHE of Malaysia.

B.T. Chan, C.W. Ong, E. Lim, N. A. Abu Osman are with Department of Biomedical Engineering, Faculty of Engineering, University of Malaya, Kuala Lumpur 50603, Malaysia. (phone: 603-7967 6871; fax: 603-7967 7661; e-mail: beetn_chan@yahoo.com).

A. Al Abed, N. H. Lovell and S. Dokos are with the Graduate School of Biomedical Engineering, the University of New South Wales, Sydney, 2052, Australia. (e-mail: s.dokos@unsw.edu.au). approaches, i.e. the geometry-prescribed method $[5,6]$ and the fluid-structure interaction method [7-9]. The geometry-prescribed CFD method uses prescribed moving boundaries to simulate movement of the cardiac wall. On the other hand, the fluid-structure interaction approach takes into account the interaction between the fluid and the structure and provides additional important parameters such as myocardial deformation and wall stress.

Although numerous models for the ventricles have been published [6-9], they have not been widely used in the study of cardiac diseases. One of the few studies include the geometry-prescribed model by Baccani et al. [5], who analyzed the vortex dynamics in an axisymmetric left ventricle with DCM during filling. It was observed that geometrical alteration of the left ventricle in DCM induces changes in the vortex patterns. In another study, Loerakker et al. [10] developed an axisymmetric fluid dynamics model of the left ventricle, coupled to a lumped parameter model of the complete circulation, to investigate the influence of a ventricular assist device on the vortex dynamics in a ventricle with dilated cardiomyopathy.

The present work investigates the effects of geometrical modification in a ventricle with DCM on the intraventricular flow distributions during the filling phase. The process of vortex ring formation arises in the filling phase and is believed to play an important role in determining the pumping efficiency during systole. We present two different models with the same initial geometry and boundary condition settings, based on the two approaches, i.e. the geometry-prescribed and fluid-structure interaction methods.

\section{METHOD}

\section{A. Model description}

The left ventricle was modeled as an axisymmetrical half truncated prolate spheroid. At the ventricular base, the mitral valve was modeled as a fixed opening orifice with a diameter of $2.5 \mathrm{~cm}$ with the aortic valve is closed throughout the filling phase. Blood is assumed to be incompressible and Newtonian, with a viscosity of $0.0035 \mathrm{~Pa}$.s and a density of $1050 \mathrm{~kg} / \mathrm{m}^{3}$. A uniform velocity profile as given in Fig. 1 is applied at the inlet to represent the mitral flow. The time-varying profile of filling phase is adapted from clinical data [5], and comprises the E wave, diastasis and $A$ wave which represents the 3 main phases in the mitral inflow.

In the following section, implementation of the two models are described.

\section{(i) Geometry-prescribed approach.}

We adapted the method proposed by Baccani et al. [5], where the wall motion is derived from the instantaneous 


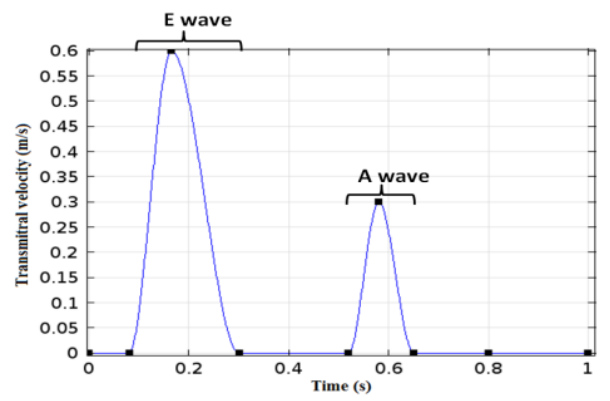

Figure 1. Inlet flow velocity profile.

blood flow rate $\mathrm{Q}(\mathrm{t})\left(\mathrm{m}^{3} / \mathrm{s}\right)$ through the mitral valve on the basis of a simple elastic membrane model. The rate of change of the radius, $\mathrm{R}(\mathrm{m})$, and the height, $\mathrm{H}(\mathrm{m})$ are:

$$
\begin{aligned}
& \frac{d R}{d t}=\frac{3 Q(t)}{\pi} \frac{2 H^{2}-R^{2}}{10 H^{3} R-4 H R^{3}} \\
& \frac{d H}{d t}=\frac{3 Q(t)}{\pi} \frac{H^{3}}{2 H^{2} R-R^{3}} \frac{2 H^{2}-R^{2}}{10 H^{3} R-4 H R^{3}}
\end{aligned}
$$

\section{(ii) Fluid-structure interaction approach}

An elastic wall with a density of $1366 \mathrm{~kg} / \mathrm{m} 3$, Poisson ratio of 0.49 and Young's modulus of $10 \mathrm{kPa}$ was used to represent the ventricle in the passive state. The wall thickness decreased along the ventricular wall from the base to the apex, with 4 $\mathrm{mm}$ at the base and $2 \mathrm{~mm}$ at the apex. The horizontal wall at the ventricular base was fixed in place while other parts of the wall were allowed to move freely. When blood filled the ventricular chamber, the wall deformed accordingly, depending on the load imposed by the fluid and the wall properties.

\section{B. Simulation of DCM}

To simulate varying degrees of DCM, different values of initial radius and height were used to achieve different end diastolic volumes (EDV) $(268 \mathrm{~mL}$ for moderate dilatation and $400 \mathrm{~mL}$ for severe dilatation). A normal ventricle with an end diastolic volume of $130 \mathrm{~mL}$ is simulated to serve as a baseline for comparison.

\section{Numerical Scheme}

The quantitative blood flow dynamics was solved using the continuity (3) and incompressible Navier-Stokes equations (4),

$$
\begin{aligned}
& \nabla \cdot \mathbf{v}=0 \\
& \rho\left(\frac{\delta \mathbf{v}}{\delta \mathrm{t}}+\mathbf{v} \cdot \nabla \mathbf{v}\right)=-\nabla \mathrm{p}+\mathbf{F}+\mu \nabla^{2} \mathbf{v}
\end{aligned}
$$

where $\mathbf{v}(\mathrm{m} / \mathrm{s})$ is fluid velocity vector, $\rho\left(\mathrm{kg} / \mathrm{m}^{3}\right)$ is fluid density, $\mathbf{p}(\mathrm{Pa})$ is pressure and $\mu$ ( $\mathrm{Pa} . \mathrm{s})$ is fluid viscosity. In the fluid-structure interaction model, the coupling between the fluid and the myocardial wall was taken into account at the fluid-solid interface through the following relationships:

$$
\begin{aligned}
& \mathbf{u}_{f}=\mathbf{u}_{s} \\
& \boldsymbol{\sigma}_{f} \cdot \mathbf{n}=-\boldsymbol{\sigma}_{s} \cdot \mathbf{n}
\end{aligned}
$$

where $\mathbf{u}(\mathrm{m})$ represents the displacements, $\boldsymbol{\sigma}(\mathrm{Pa})$ represents the stress tensors, subscript $f$ and $s$ represent fluid and solid at the fluid solid interface, and $\mathbf{n}$ represents the unit normal vector.

\section{RESULTS AND DISCUSSION}

\section{A. Geometry-prescribed implementation}

The vortex formation and movement patterns in our simulations generally agree with that shown in the published literature [5, 7] (not shown).Comparing the three different cardiac conditions, the normal ventricle has the greatest vortex strength (maximum strength of $0.04378 \mathrm{~m}^{2} / \mathrm{s}$ ) as compared to the moderately (maximum strength of $0.03782 \mathrm{~m}^{2} / \mathrm{s}$ ) and severely dilated ventricle (maximum strength of 0.03513 $\mathrm{m}^{2} / \mathrm{s}$ ), as shown in Fig. 2. This agrees with data from published simulations [10] and experimental results [11], which provided quantitative vortex measurements. The vorticity magnitude shown in Fig. 2 was contributed by both the primary (first peak) and secondary vortices (second peak), as well as the growing boundary layer. A high vorticity magnitude helps the propagation of the vortex ring towards the apex. Due to the weaker vortex strength in a more dilated ventricle, the vortex ring is propelled towards the apex by weaker convective acceleration and thus has less energy. As a result, vortex washout is reduced, leading to the stagnation of the apical flow region. In an experimental study, Maze et al. [12] reported that thrombus formation was commonly found in dilated ventricles associated with cardiomyopathy.

On the other hand, we also analyzed intraventricular pressure gradients (IVPGs), i.e. pressure difference between the base and the apex of the ventricle, in the three different cardiac conditions. An increase in IVPGs is believed to improve systolic and diastolic function through an increase in left ventricular suction and elastic recoil [13]. Our results showed that IVPGs decreased in more dilated ventricle (Fig. 3 ), with slower propagation, as reported by Yotti et al [14]. This reduces the capability of the ventricle to generate suction, leading to an impaired filling.

\section{B. FSI implementation}

Generally, the results obtained from the FSI implementation shows a good agreement with the geometry-prescribed approach. Figs. 4 and 5 show a comparison of the vortex distribution in the ventricle under the three different cardiac conditions. In both simulations, the propagating vortex ring in dilated ventricles has less interaction with the ventricular wall, thereby producing a thinner boundary layer. As expected in both approaches, vortex strength in the more dilated ventricles is lower as compared to the healthy condition.

The most notable difference between the two implementations lies in the vortex attachment to the edge of the mitral orifice. In the FSI implementation results, the vortex ring was attached to the edge of the mitral orifice for a longer period. In addition, a thinner boundary layer was observed, and less interaction occurs between the vortex and the wall Dissimilar with the geometry-prescribed method, wall expansion of the FSI model depends on the fluid force exerted on it.

Furthermore, due to the fixed constraint at the basal wall, there is a slight difference between the two methods in regards to the geometry, especially at the basal region of the ventricle, which leads to the difference in the result. 


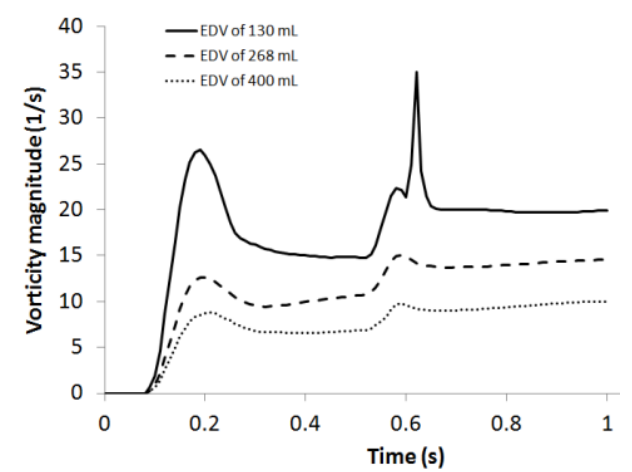

Figure 2. Vorticity magnitude in the ventricle with EDV of: $130 \mathrm{~mL}$ (normal), $268 \mathrm{~mL}$ (moderately dilated) and $400 \mathrm{~mL}$ (severely dilated).

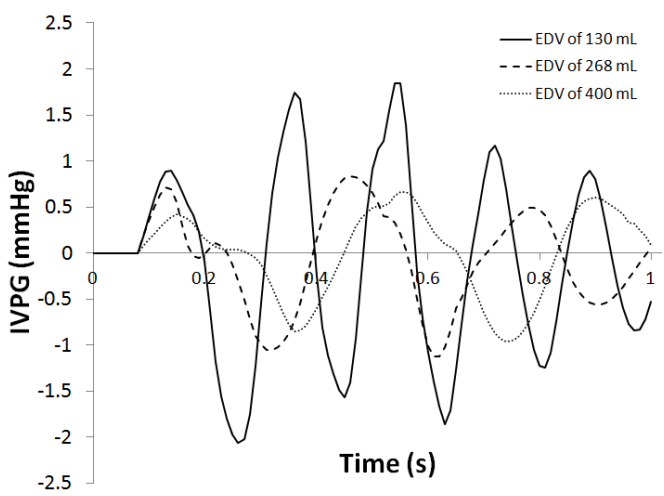

Figure 3. IVPGs in the ventricle with EDV of: $130 \mathrm{~mL}$ (normal), $268 \mathrm{~mL}$ (moderately dilated) and $400 \mathrm{~mL}$ (severely dilated).

Compared to the geometry-prescribed method, FSI approach provides additional parameters such as myocardial displacement and wall stress, which serve as useful prognostic determinants of heart disease. An increase in wall stress increases the risk for arrhythmia and energy metabolism, which forces the dilated ventricle to work even harder. The wall movement depends on the wall properties and the fluid force exerted on it. Fig. 6 shows that the wall displacement and velocity were significantly reduced by ventricular dilatation at the same inflow volume and wall properties. The decreasing wall velocity indicated loss of elastic recoil energy which contribute to impaired diastolic suction and contractile function for blood ejection during systole $[14,15]$.

On the other hand, myocardium stress is primarily determined by the wall properties and total myocardial displacement. Since identical wall properties were imposed for the three cardiac conditions in the present study, ventricular wall stress depends only on the wall displacement. As a result, higher wall stress was found in the normal ventricle compared to that for the dilated cases in the present study (Fig. 7). This contradicts with the published experimental studies which showed an increase in wall stress under the DCM condition $[16,17]$. This can be explained by the fact that same ventricular wall properties (Young's modulus) were imposed on all cardiac conditions in the present study. Generally, a patient with DCM has a stiffer ventricle compared to that of a normal subject. The reduction of wall compliance is the key factor which causes an increase in wall stress in the DCM condition. To more realistically reproduce DCM in the future, different myocardial wall properties have to be used for different cardiac conditions.

\section{Model limitations}

In the present study, the left ventricle was modeled using the axisymmetrical approach. As a result, we were not able to simulate the asymmetric vortex profile occurring in the ventricle. Schenkel et al. [9] suggested that the asymmetry in the vortex development helps to avoid a stagnant area in the ventricular apex, thereby improving the pumping efficiency.

A lower velocity magnitude (peak velocity of $0.6 \mathrm{~m} / \mathrm{s}$ instead of $1 \mathrm{~m} / \mathrm{s}$ normally occurring in a healthy ventricle) was imposed at the inlet boundary in all simulations due to a convergence issue at higher velocities. This lower velocity magnitude does not affect the vortex patterns, but decreases the overall propagating velocity of the vortex.

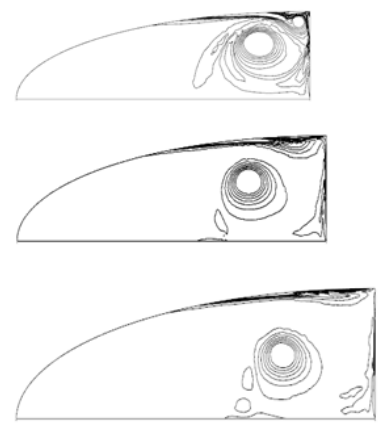

Figure 4. Vorticity in the ventricle with EDV: (top) $130 \mathrm{~mL}$; (middle) $268 \mathrm{~mL}$; and (bottom) $400 \mathrm{~mL}$ at $\mathrm{t}=0.36 \mathrm{~s}$ in the geometry-prescribed implementation.
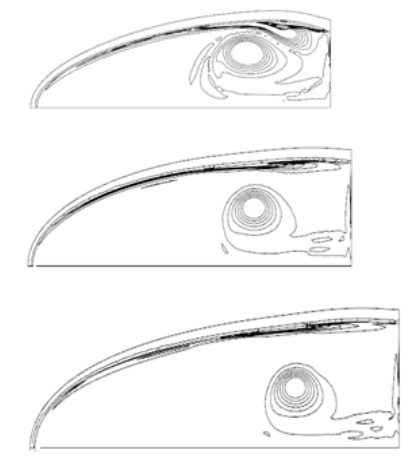

Figure 5. Vorticity in the ventricle with EDV: (top) $130 \mathrm{~mL}$; (middle) $268 \mathrm{~mL}$ and (bottom) $400 \mathrm{~mL}$ at $\mathrm{t}=0.36 \mathrm{~s}$ in the FSI-implementation.

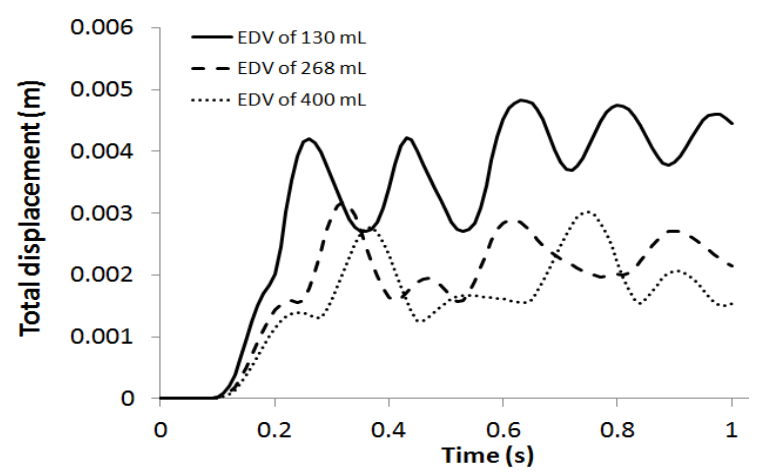

Figure 6. Total displacement of ventricular wall (fluid structure boundary) of ventricle with EDV of: $130 \mathrm{~mL}$ (normal), $268 \mathrm{~mL}$ (moderately dilated) and $400 \mathrm{~mL}$ (severely dilated). 


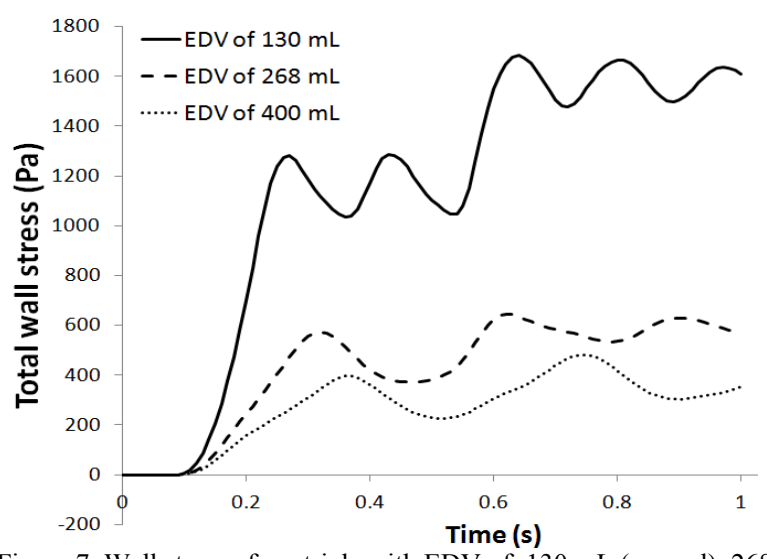

Figure 7. Wall stress of ventricle with EDV of: $130 \mathrm{~mL}$ (normal), $268 \mathrm{~mL}$ (moderately dilated) and $400 \mathrm{~mL}$ (severely dilated).

Clinically, a DCM ventricle has a thinner wall compared to that of a healthy ventricle. Apart from that, impaired and restrictive filling pattern has been observed to occur in early and late stage DCM patients. Since the main aim of the present study is to investigate the effects of geometrical modification on the flow pattern, we have used identical wall thickness and filling velocity in all our simulations. For simplification purpose, we have also fixed the ventricular base and mitral valve radius to allow the same amount of volume to be injected into the ventricle during diastole.

In addition, significant oscillations were observed in the temporal profile of wall displacement and stress, due to the sloshing of fluid within the ventricular cavity (Figs. 6 and 7). In future, these oscillations could be reduced by using a viscoelastic material description of the myocardium, or by incorporating an aortic valve outlet to alleviate the ventricular pressure and dampen the oscillations.

\section{CONCLUSION}

The present work investigates the effects of geometrical modification in a ventricle on the intraventricular flow distributions using two different models, i.e. the geometry-prescribed and fluid-structure interaction approaches. The simulated vortex patterns and IVPGs agree with those reported in the literature, with weaker vortex strength and smaller IVPGs observed in more dilated ventricles. Although similar simulation results were obtained from the two methods, FSI approach provides additional information such as wall stress and strain, which are important predictors of ventricular function. Moreover, cardiac diseases can be more easily and realistically modeled compared to the geometry-prescribed method, which may involve more complicated equations and assumptions that often results in convergence problem.

\section{ACKNOWLEDGMENT}

This study was funded by Ministry of Higher Education (MOHE) of Malaysia, grant number UM.C/HIR/MOHE/ENG/14 D000014-16001 and supported by University of Malaya Faculty of Engineering.

\section{REFERENCES}

[1] J. L. Jefferies, and J. A. Towbin, "Dilated Cardiomyopathy," Lancet, vol. 375, no. 9716, pp. 752-762, 2010.

[2] Augst D, Barratt D, Hughes A, Glor F, Thom S, and X. X, "Accuracy and reproducibility of CFD predicted wall shear stress using $3 \mathrm{D}$ ultrasound images," ASME J. Biomed. Eng., vol. 125, pp. 218-222, 2003.

[3] S. Beppu, S. Izumi, K. Miyatake, S. Nagata, Y. D. Park, H. Sakakibara, and Y. Nimura, "Abnormal blood pathways in left ventricular cavity in acute myocardial infarction. Experimental observations with special reference to regional wall motion abnormality and hemostasis," Circulation, vol. 78, pp. 157-164, 1988.

[4] O. Pierrakos, and P. P. Vlachos, "The Effect of Vortex formation on Left Ventricualr Filling and Mitral Valve Efficiency," Journal of Biomechanical Engineering, vol. 128, pp. 527-539, 2006.

[5] B. Baccani, F. Domenichini, G. Pedrizzetti, and G. Tonti, "Fluid dynamics of the left ventricular filling in dilated cardiomyopathy," Journal of Biomechanics, vol. 35, pp. 665-671, 2002.

[6] J. A. Vierendeels, K. Riemslagh, and E. Dick, "Computer simulation of intraventricular flow and pressure gradients during diastole," Journal of Biomechanical Engineering, vol. 122, pp. 667-674, 2000.

[7] Y. G. Cheng, H. Oertel, and T. Schenkel, "Fluid-Structure Coupled CFD Simulation of the Left Ventrivular Flow During Filling Phase," Annals of Biomedical Engineering, vol. 33, no. 5, pp. 567-576, 2005.

[8] H. Watanabe, T. Hisada, S. Sugiura, J. Okada, and H. Fukunari, "Computer Simulation of Blood Flow, Left Ventricular Wall Motion and Their Interrelationship by Fluid-Structure Interaction Finite Element Method," JSME International Journal, vol. 45, no. 4, pp. 1003-1011, 2002.

[9] T. Schenkel, M. Malve, M. Reik, M. Markl, B. Jung, and H. Oertel, "MRI-Based CFD Analysis of Flow in a Human Left Ventricle Methodology and Application to a Healthy Heart," Annals of Biomedical Engineering, vol. 37, no. 3, pp. 503-515, 2008.

[10] S. Loerakker, L. G. E. Cox, G. J. F. van Heijst, B. A. J. M. de Mol, and F. N. van de Vosse, "Influence of dilated cardiomyopathy and a left ventricular assist device on vortex dynamics in the left ventricle," Computer Methods in Biomechanics and Biomedical Engineering, vol. 11, no. 6, pp. 649-660, 2008

[11] G. R. Hong, G. Pedrizzetti, G. Tonti, P. Li, Z. Wei, J. K. Kim, A. Baweja, S. Liu, N. Chung, H. Houle, J. Narula, and M. A. Vannan, "Characterization and Quantification of Vortex Flow in the Human Left Ventricle by Contrast Echocardiography Using Vector Particle Image Velocimetry," JACC, vol. 1, no. 6, pp. 705-717, 2008.

[12] S. Maze, M. Kotler, and W. Parry, "Flow characteristics in the dilated left ventricle with thrombus: qualitative and quantitative Doppler analysis," J Am Coll Cardiol, vol. 13, no. 4, pp. 873-881, March 15, $1989,1989$.

[13] M. S. Firstenberg, N. G. Smedira, N. L. Greenberg, D. L. Prior, P. M. McCarthy, M. J. Garcia, and D. J. Thomas, "Relationship Between Early Diastolic Intraventricualr pressure Gradients, an Index of Elastic Recoil, and Improvements in Systolic and Diastolic Function," Circulation, vol. 104, no. suppI I, pp. I-330-335, 2001.

[14] R. Yotti, J. Bermejo, J. C. Antoranz, M. M. Desco, C. Cortina, J. L. Rojo-Alvarez, C. Allue, L. Martin, M. Moreno, J. A. Serrano, R. Munoz, and M. A. Garcia-Fernandez, "A Noninvasive Method for Assesing Impaired Diastolic Suction in Patients With Dilated Cardiomyopathy," Circulation, vol. 112, pp. 2921-2929, 2005.

[15] E. H. Sonnenblick, "The structural basis and importance of restoring forces and elastic recoil for the filling of the heart," European Heart Journal, vol. 1, no. Supplement A, pp. 107-110, 1980.

[16] W. Hayashida, T. Kumada, R. Nohara, H. Tanio, M. Kambayashi, N. Ishikawa, Y. Nakamura, Y. Himura, and C. Kawai, "Left Ventricular Regional Wall Stress in Dilated Cardiomyopathy," Circulation, vol. 82, no. 6, pp. 2075-83, 1990.

[17] W. Grossman, L. P. McLaurin, S. P. Moos, M. Stefadouros, and D. T. Young, "Wall Thickness and Diastolic Properties of the left Ventricle," Circulation, vol. XLIX, pp. 129-135, 1974. 